\title{
SUPPLEMENTARY MATERIAL: Sequential Monte Carlo Samplers with Independent Markov chain Monte Carlo Proposals
}

\author{
L. F. South ${ }^{* \dagger, \ddagger}$, A. N. Pettitt ${ }^{*, \dagger}$, and C. C. Drovandi ${ }^{*, \dagger, \uparrow}$
}

\section{Appendix A: ESS after Recycling}

Assume that $T+1$ weighted samples target the same distribution, and the $t$-th weighted sample has an ESS of $\mathrm{ESS}_{t}$ for $t=0, \ldots, T$. This situation applies when populations $\left\{\boldsymbol{\theta}_{t}^{i}\right\}_{i=1}^{N}$ targeting $\pi_{t}$ for $t=0, \ldots, T$ have been reweighted to target the posterior $\pi_{T}$ in likelihood annealing SMC. If these multiple weighted samples are combined together based on the sample weight $\lambda_{t}=\mathrm{ESS}_{t} / \sum_{l=0}^{T} \mathrm{ESS}_{l}$, then the total ESS targeting the posterior is exactly the sum of the ESS from each sample. This is shown below using the notation from the CISPP recycling method, but the same concepts apply to the CIS $_{\text {IP }}$ recycling method.

$$
\begin{aligned}
\mathrm{ESS} & =\frac{1}{\sum_{t=0}^{T} \sum_{i=1}^{N_{t}}\left(\tilde{K}_{t}^{i}\right)^{2}} \\
& =\frac{1}{\sum_{t=0}^{T} \sum_{i=1}^{N_{t}}\left(\lambda_{t} K_{t}^{i}\right)^{2}} \\
& =\frac{1}{\sum_{t=0}^{T}\left\{\lambda_{t}^{2} \sum_{i=1}^{N_{t}}\left(K_{t}^{i}\right)^{2}\right\}} \\
& =\frac{1}{\sum_{t=0}^{T} \lambda_{t}^{2} \cdot \frac{1}{\operatorname{ESS}_{t}}} \\
& =\frac{1}{\sum_{t=0}^{T}\left(\frac{\mathrm{ESS}_{t}}{\sum_{l=0}^{T} \mathrm{ESS}_{l}}\right)^{2} \cdot \frac{1}{\operatorname{ESS}_{t}}} \\
& =\frac{\left(\sum_{l=0}^{T} \operatorname{ESS}_{l}\right)^{2}}{\sum_{t=0}^{T} \mathrm{ESS}_{t}} \\
& =\sum_{t=0}^{T} \mathrm{ESS}_{t} .
\end{aligned}
$$

* School of Mathematical Sciences, Queensland University of Technology, Australia

${ }^{\dagger}$ ARC Centre of Excellence for Mathematical \& Statistical Frontiers (ACEMS)

${ }^{\ddagger}$ Supported by an Australian Government Research Training Program Scholarship and by ACEMS

§upported by Australian Research Council's Discovery Project funding scheme DP110100159

`Supported by Australian Research Council's Discovery Early Career Researcher Award funding scheme DE160100741 


\section{Appendix B: Mixture Model Proposal Density}

This appendix shows how to compute the density for our independent copula mixture proposals. The MGMM is fitted for particles where the marginals are roughly standard normal and a candidate on this scale is denoted $\boldsymbol{X}^{*}$. The proposal density for the candidate $\boldsymbol{X}^{*}$ is

$$
f\left(\boldsymbol{X}^{*} \mid \hat{\boldsymbol{c}}, \hat{\boldsymbol{\mu}}, \hat{\boldsymbol{\Sigma}}\right)=\sum_{k=1}^{K} \hat{c}_{k} \mathcal{N}\left(\boldsymbol{X}^{*} ; \hat{\boldsymbol{\mu}}_{k}, \hat{\boldsymbol{\Sigma}}_{k}\right) .
$$

However, this candidate is on the transformed scale so the proposal density must be adjusted using the Jacobian of the transformations. The first step in transforming $\boldsymbol{X}^{*}$ to a candidate $\boldsymbol{\theta}^{*}$ is to transform the candidate to be on the approximately $\mathcal{U}(0,1)$ space using the cumulative distribution function of the standard normal distribution. The candidate on the new scale is then $\boldsymbol{U}^{*}[j]=\Phi\left(\boldsymbol{X}^{*}[j]\right)$ for $j=1, \ldots, p$ and the new proposal density is determined by the following form,

$$
\begin{aligned}
g\left(\boldsymbol{U}^{*} \mid \hat{\boldsymbol{c}}, \hat{\boldsymbol{\mu}}, \hat{\boldsymbol{\Sigma}}\right) & =f\left(\boldsymbol{X}^{*} \mid \hat{\boldsymbol{c}}, \hat{\boldsymbol{\mu}}, \hat{\boldsymbol{\Sigma}}\right)\left|\frac{d \boldsymbol{X}}{d \boldsymbol{U}}\right| \\
& =f\left(\boldsymbol{X}^{*} \mid \hat{\boldsymbol{c}}, \hat{\boldsymbol{\mu}}, \hat{\boldsymbol{\Sigma}}\right) \prod_{j=1}^{p} \frac{1}{\mathcal{N}\left(\boldsymbol{X}^{*}[j], 0,1\right)}
\end{aligned}
$$

Next, the quantile function of the marginals are used to transform the candidate $\boldsymbol{U}^{*}$ to $\boldsymbol{\theta}^{*}$, such that $\boldsymbol{\theta}^{*}[j]=Q_{j}^{\hat{\boldsymbol{\eta}}_{j}}\left(\boldsymbol{U}^{*}[j]\right)$ for $j=1, \ldots, p$. The final proposal density for $\boldsymbol{\theta}^{*}$ with fitted parameters $\phi_{t}=(\hat{\boldsymbol{\eta}}, \hat{\boldsymbol{c}}, \hat{\boldsymbol{\mu}}, \hat{\boldsymbol{\Sigma}})$ is then

$$
\begin{aligned}
q^{\phi_{t}\left(\boldsymbol{\theta}^{*}\right)} & =g\left(\boldsymbol{U}^{*} \mid \hat{\boldsymbol{c}}, \hat{\boldsymbol{\mu}}, \hat{\boldsymbol{\Sigma}}\right)\left|\frac{d \boldsymbol{U}}{d \boldsymbol{\theta}}\right| \\
& =g\left(\boldsymbol{U}^{*} \mid \hat{\boldsymbol{c}}, \hat{\boldsymbol{\mu}}, \hat{\boldsymbol{\Sigma}}\right) \prod_{j=1}^{p} g_{j}^{\hat{\boldsymbol{\eta}}_{j}}\left(\boldsymbol{\theta}^{*}[j]\right) \\
& =\left\{\prod_{j=1}^{p} \frac{1}{\mathcal{N}\left(\boldsymbol{X}^{*}[j] ; 0,1\right)}\right\}\left\{\prod_{j=1}^{p} g_{j}^{\hat{\boldsymbol{\eta}}_{j}}\left(\boldsymbol{\theta}^{*}[j]\right)\right\} \sum_{k=1}^{K} \hat{c}_{k} \mathcal{N}\left(\boldsymbol{X}^{*} ; \hat{\boldsymbol{\mu}}_{k}, \hat{\boldsymbol{\Sigma}}_{k}\right) .
\end{aligned}
$$

\section{Appendix C: Factor Analysis Example: Additional Figures}

Posterior estimates for the marginals are shown in Figures 1 for the one factor model, 2 for the two factor model and 3 for the three factor model. Efficiency for the univariate posterior medians in the one, two and three factor models can be seen in Tables 1, 2 and 3, respectively. The bivariate distributions for the three factor model are extremely complex and these can be seen in Figure 4. 


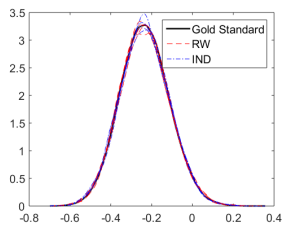

(a) $\log \Lambda_{11}$

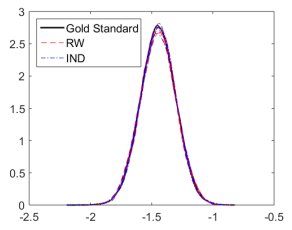

(e) $\log \Lambda_{55}$

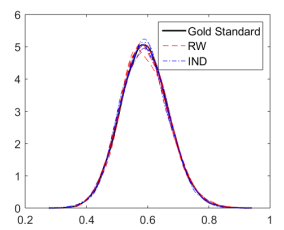

(i) $\beta_{31}$

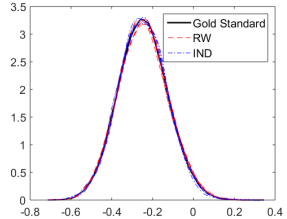

(b) $\log \Lambda_{22}$

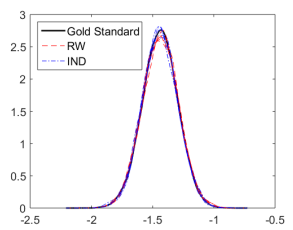

(f) $\log \Lambda_{66}$

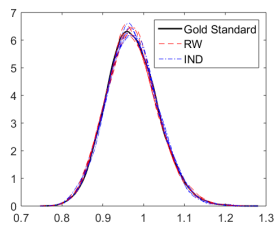

(j) $\beta_{41}$

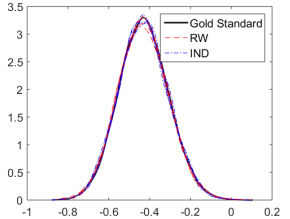

(c) $\log \Lambda_{33}$

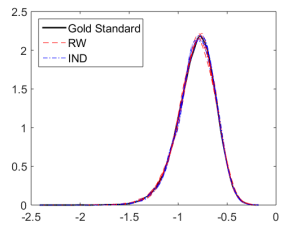

(g) $\log \beta_{11}$

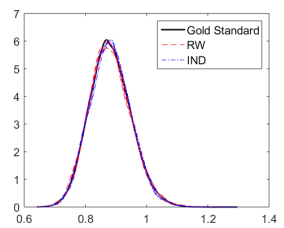

(k) $\beta_{51}$

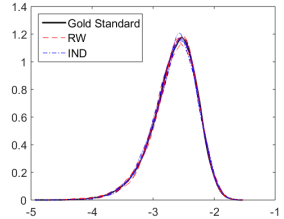

(d) $\log \Lambda_{44}$

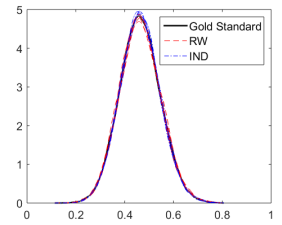

(h) $\beta_{21}$

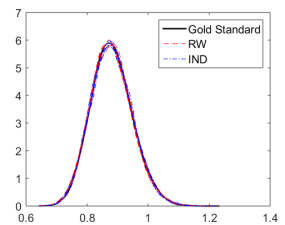

(1) $\beta_{61}$

Figure 1: FA example: Posterior estimates for the marginals of the one factor model based on a single gold standard run (solid), five runs with the RW kernel (dash) and five runs with the IND kernel (dot-dash).

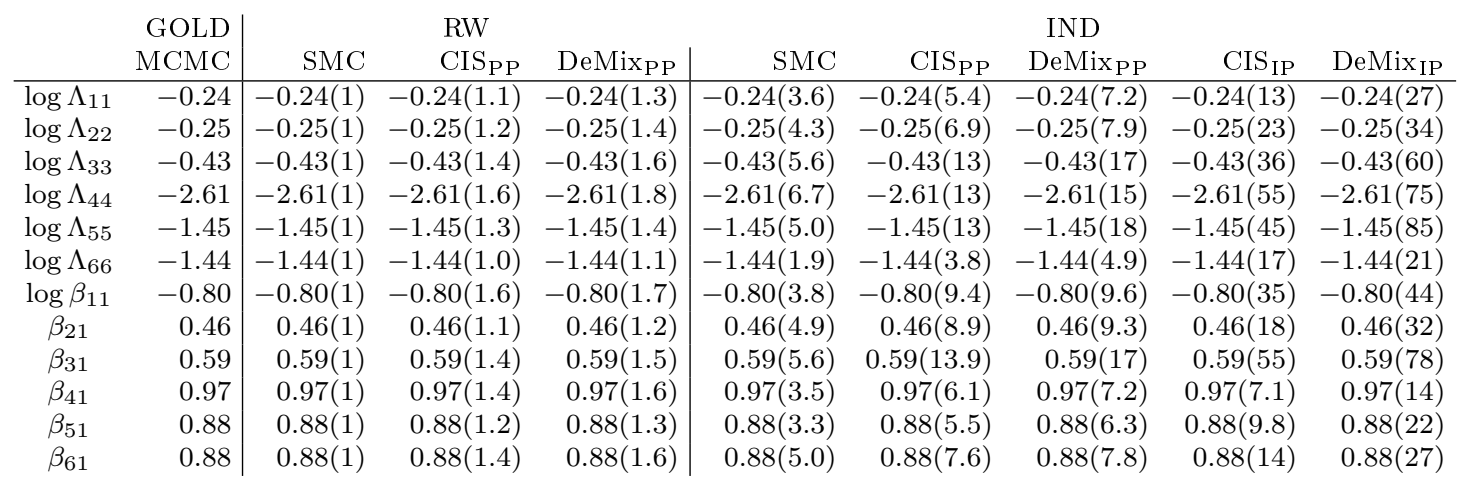

Table 1: FA example: Posterior median estimates for the one factor model with efficiency in parentheses, based on $100 \mathrm{SMC}$ runs 


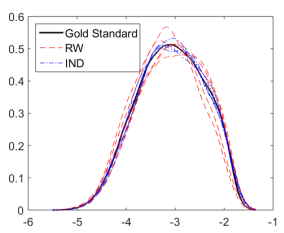

(a) $\log \Lambda_{11}$

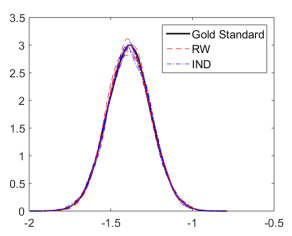

(e) $\log \Lambda_{55}$

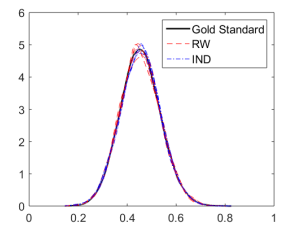

(i) $\beta_{31}$

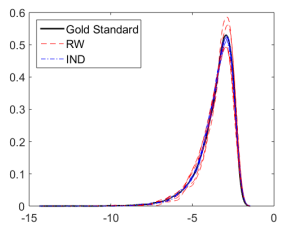

(m) $\log \beta_{22}$

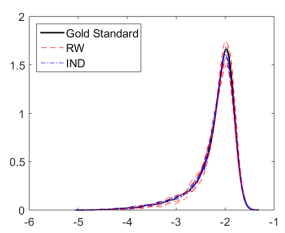

(b) $\log \Lambda_{22}$

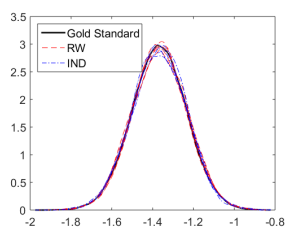

(f) $\log \Lambda_{66}$

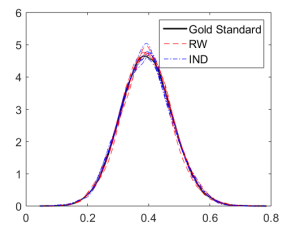

(j) $\beta_{41}$

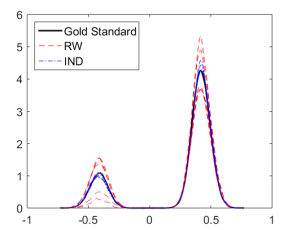

(n) $\beta_{32}$

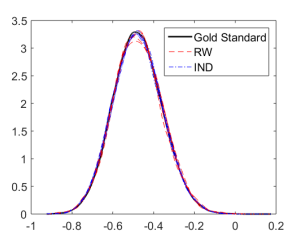

(c) $\log \Lambda_{33}$

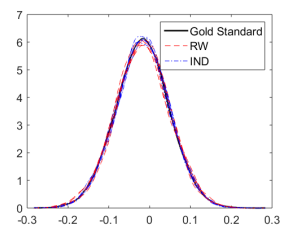

(g) $\log \beta_{11}$

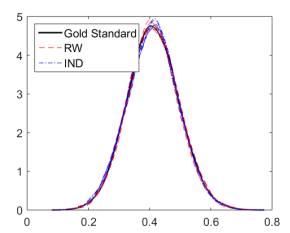

(k) $\beta_{51}$

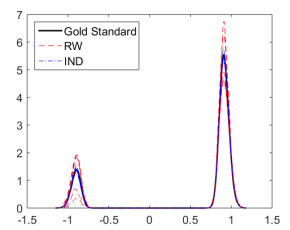

(o) $\beta_{42}$

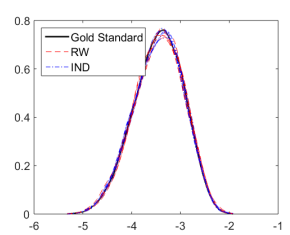

(d) $\log \Lambda_{44}$

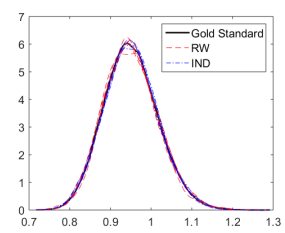

(h) $\beta_{21}$

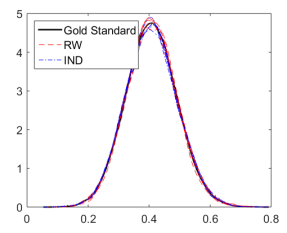

(l) $\beta_{61}$

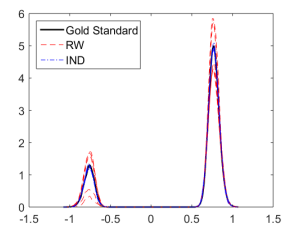

(p) $\beta_{52}$

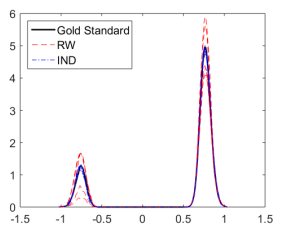

(q) $\beta_{62}$

Figure 2: FA example: Posterior estimates for the marginals of the two factor model based on a single gold standard run (solid), five runs with the RW kernel (dash) and five runs with the IND kernel (dot-dash). 


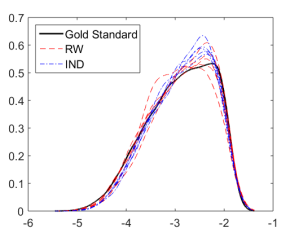

(a) $\log \Lambda_{11}$

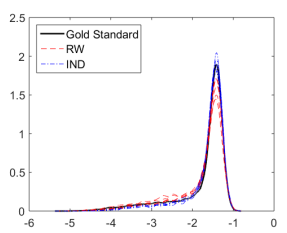

(e) $\log \Lambda_{55}$

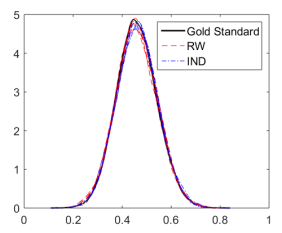

(i) $\beta_{31}$

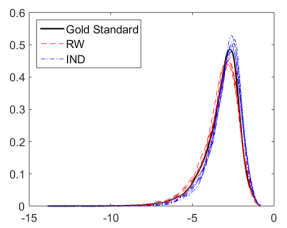

(m) $\log \beta_{22}$

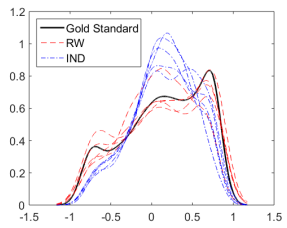

(q) $\beta_{62}$

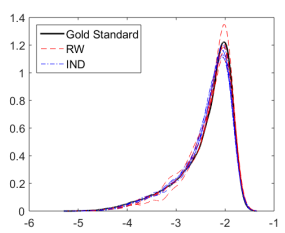

(b) $\log \Lambda_{22}$

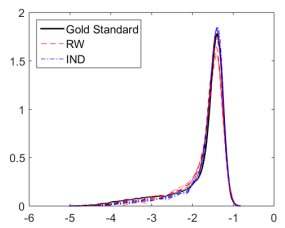

(f) $\log \Lambda_{66}$

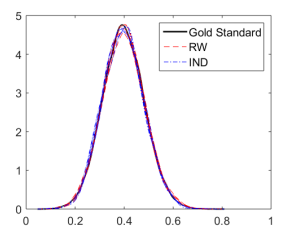

(j) $\beta_{41}$

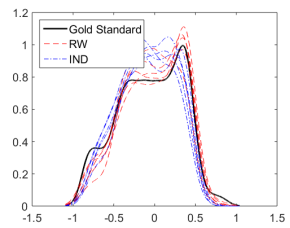

(n) $\beta_{32}$

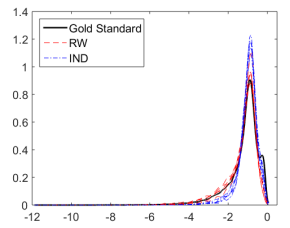

(r) $\log \beta_{33}$

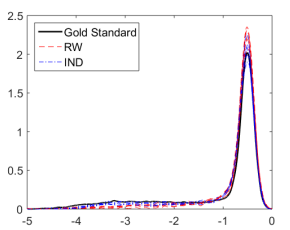

(c) $\log \Lambda_{33}$

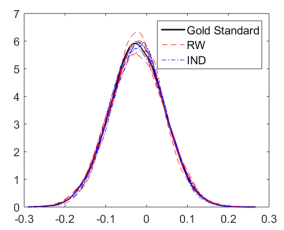

(g) $\log \beta_{11}$

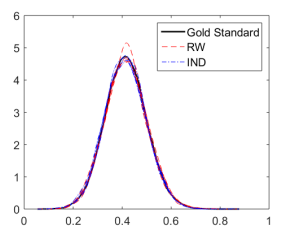

(k) $\beta_{51}$

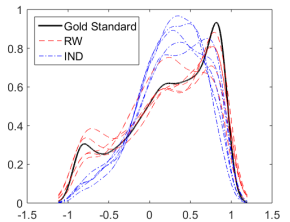

(o) $\beta_{42}$

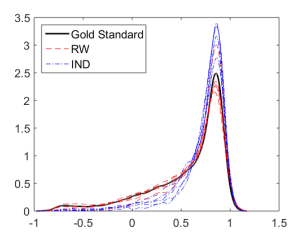

(s) $\beta_{43}$

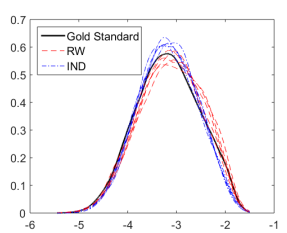

(d) $\log \Lambda_{44}$

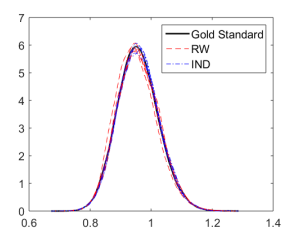

(h) $\beta_{21}$

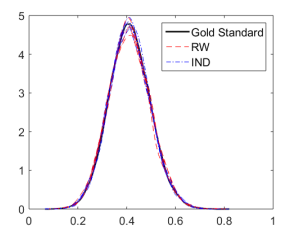

(l) $\beta_{61}$

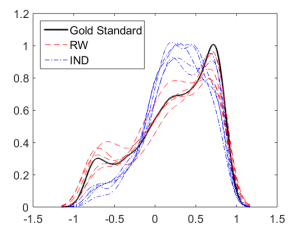

(p) $\beta_{52}$

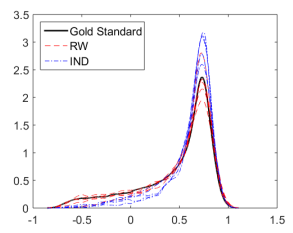

(t) $\beta_{53}$

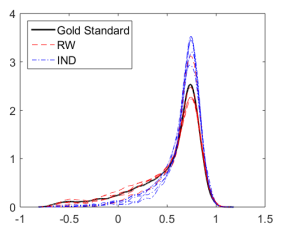

(u) $\beta_{63}$

Figure 3: FA example: Posterior estimates for the marginals of the three factor model based on a single gold standard run (solid), five runs with the RW kernel (dash) and five runs with the IND kernel (dot-dash). 


\begin{tabular}{|c|c|c|c|c|c|c|c|c|c|}
\hline \multirow{2}{*}{\multicolumn{2}{|c|}{ GOLD }} & \multicolumn{3}{|c|}{ RW } & \multicolumn{5}{|c|}{ IND } \\
\hline & & SMC & $\mathrm{CIS}_{\mathrm{PP}}$ & $\operatorname{DeMix}_{\mathrm{PP}}$ & SMC & $\mathrm{CIS}_{\mathrm{PP}}$ & $\operatorname{DeMix}_{\mathrm{PP}}$ & $\mathrm{CIS}_{\mathrm{IP}}$ & DeMix $_{I P}$ \\
\hline $\log \Lambda_{11}$ & -3.06 & $-3.03(1)$ & $-3.03(0.96)$ & $-3.03(0.97)$ & $-3.06(270)$ & $-3.05(260)$ & $-3.05(300)$ & $-3.06(850)$ & $-3.06(1,200)$ \\
\hline $\log \Lambda_{22}$ & -2.08 & $-2.09(1)$ & $-2.09(0.98)$ & $-2.09(1.0)$ & $-2.08(250)$ & $-2.09(260)$ & $-2.09(300)$ & $-2.08(700)$ & $-2.08(950)$ \\
\hline $\log \Lambda_{33}$ & -0.48 & $-0.48(1)$ & $-0.48(0.79)$ & $-0.48(0.80)$ & $-0.48(8.5)$ & $-0.48(18)$ & $-0.48(18)$ & $-0.48(34)$ & $-0.48(62)$ \\
\hline $\log \Lambda_{44}$ & -3.42 & $-3.43(1)$ & $-3.42(0.91)$ & $-3.42(0.90)$ & $-3.42(13)$ & $-3.42(15)$ & $-3.42(16)$ & $-3.43(31)$ & $-3.43(48)$ \\
\hline $\log \Lambda_{55}$ & -1.39 & $-1.39(1)$ & $-1.39(0.98)$ & $-1.39(1.1)$ & $-1.39(8.1)$ & $-1.39(14)$ & $-1.39(15)$ & $-1.39(27)$ & $-1.39(28)$ \\
\hline $\log \Lambda_{66}$ & -1.37 & $-1.37(1)$ & $-1.37(0.92)$ & $-1.37(0.92)$ & $-1.37(10)$ & $-1.37(15)$ & $-1.37(13)$ & $-1.37(24)$ & $-1.37(38)$ \\
\hline $\log \beta_{11}$ & -0.02 & $-0.02(1)$ & $-0.02(0.96)$ & $-0.02(1.0)$ & $-0.02(22)$ & $-0.02(25)$ & $-0.02(21)$ & $-0.02(100)$ & $-0.02(150)$ \\
\hline$\beta_{21}$ & 0.95 & $0.95(1)$ & $0.95(1.0)$ & (1.0) & $0.95(33)$ & $0.95(33)$ & $0.95(26)$ & $0.95(100)$ & $0.95(120)$ \\
\hline$\beta_{31}$ & 0.45 & $0.45(1)$ & $0.45(0.81)$ & $0.45(0.83)$ & $0.45(6.6)$ & $0.45(14)$ & $0.45(16)$ & $0.45(21)$ & $0.45(35)$ \\
\hline$\beta_{41}$ & 0.39 & $0.39(1)$ & $0.39(1.1)$ & $0.39(1.1)$ & $0.39(52)$ & $0.39(120)$ & $0.39(120)$ & $0.39(170)$ & $0.39(280)$ \\
\hline$\beta_{51}$ & 0.41 & $0.41(1)$ & $0.41(1.0)$ & $0.41(1.0)$ & $0.41(38)$ & $0.41(88)$ & $0.41(96)$ & $0.41(120)$ & $0.41(180)$ \\
\hline$\beta_{61}$ & 0.41 & $0.41(1)$ & $0.41(1.1)$ & $0.41(1.1)$ & $0.41(30)$ & $0.41(58)$ & $0.41(56)$ & $0.41(91)$ & $0.41(150)$ \\
\hline $\log \beta_{22}$ & -3.30 & $-3.33(1)$ & $-3.33(0.99)$ & $-3.33(0.98)$ & $-3.29(220)$ & $-3.28(240)$ & $-3.28(280)$ & $-3.30(1,200)$ & $-3.30(1,800)$ \\
\hline$\beta_{32}$ & 0.40 & $0.36(1)$ & $0.36(1.0)$ & $0.36(1.0)$ & $0.40(11,000)$ & $0.40(8,600)$ & $0.40(7,500)$ & $0.40(130,000)$ & $0.40(180,000)$ \\
\hline$\beta_{42}$ & 0.89 & $0.82(1)$ & $0.82(1.0)$ & $0.82(1.0)$ & $0.90(97,000)$ & $0.90(75,000)$ & $0.90(64,000)$ & $0.89(930,000)$ & $0.89(1,300,000)$ \\
\hline$\beta_{52}$ & 0.75 & $0.69(1)$ & $0.69(1.0)$ & $0.69(1.0)$ & $0.75(60,000)$ & $0.75(41,000)$ & $0.75(36,000)$ & $0.75(560,000)$ & $0.75(640,000)$ \\
\hline$\beta_{62}$ & 0.75 & $0.69(1)$ & $0.69(1.0)$ & $0.69(1.0)$ & $0.75(49,000)$ & $0.75(39,000)$ & $0.75(34,000)$ & $0.75(520,000)$ & $0.75(690,000)$ \\
\hline
\end{tabular}

Table 2: FA example: Posterior median estimates for the two factor model with efficiency in parentheses, based on 100 SMC runs

\begin{tabular}{|c|c|c|c|c|c|c|c|c|c|}
\hline & \multicolumn{3}{|c|}{ RW } & \multicolumn{5}{|c|}{ IND } \\
\hline & MCMC & $\mathrm{SMC}$ & $\mathrm{CIS}_{\mathrm{PP}}$ & $\operatorname{DeMix}_{P P}$ & SMC & CIS $_{P P}$ & $\operatorname{DeMix}_{\mathrm{PP}}$ & CIS $_{I P}$ & DeMix $_{I P}$ \\
\hline $\log \Lambda_{11}$ & -2.82 & $-2.81(1)$ & $-2.80(0.66)$ & $-2.80(0.65)$ & $-2.79(42)$ & $-2.79(36)$ & $-2.79(35)$ & $-2.80(27)$ & $-2.81(4.0)$ \\
\hline $\log \Lambda_{22}$ & -2.21 & $-2.22(1)$ & $-2.22(0.59)$ & $-2.23(0.54)$ & $-2.23(22)$ & $-2.23(21)$ & $-2.23(21)$ & $-2.22(28)$ & $-2.21(9.1)$ \\
\hline $\log \Lambda_{33}$ & -0.59 & $-0.56(1)$ & $-0.56(0.89)$ & $-0.56(0.87)$ & $-0.58(78)$ & $-0.58(61)$ & $-0.58(57)$ & $-0.58(40)$ & $-0.60(0.96)$ \\
\hline $\operatorname{gg} \Lambda_{44}$ & -3.18 & $-3.14(1)$ & $-3.14(0.78)$ & $-3.14(0.79)$ & $-3.18(100)$ & $-3.18(110)$ & $-3.18(110)$ & $-3.16(56)$ & $-3.19(4.9)$ \\
\hline $\operatorname{gg} \Lambda_{55}$ & -1.50 & $-1.52(1)$ & $-1.53(0.76)$ & $-1.53(0.74)$ & $-1.49(120)$ & $-1.49(120)$ & $-1.48(100)$ & $-1.50(130)$ & $-1.49(22)$ \\
\hline $\log \Lambda_{66}$ & -1.49 & $-1.52(1)$ & $-1.52(0.54)$ & $-1.52(0.47)$ & $-1.48(160)$ & $-1.48(130)$ & $-1.48(120)$ & $-1.50(95)$ & $-1.50(3.8)$ \\
\hline $\log \beta_{11}$ & -0.02 & $-0.02(1)$ & $-0.02(0.62)$ & $-0.02(0.66)$ & $-0.02(43)$ & $-0.02(31)$ & $-0.02(25)$ & $-0.02(7.7)$ & $-0.02(1.4)$ \\
\hline$\beta_{21}$ & 0.95 & $0.96(1)$ & $0.96(0.52)$ & 0.96 & 0.96 & $0.96(17)$ & 0.96 & $0.96(9.2)$ & $1.6)$ \\
\hline$\beta_{31}$ & 0.46 & $0.46(1)$ & $0.46(0.50)$ & 0.46 & 0.46 & 0.4 & 0.46 & $0.46(8.0)$ & $0.46(0.61)$ \\
\hline$\beta_{41}$ & 0.40 & $0.40(1)$ & $0.40(0.5$ & 0.4 & 0.39 & 0.4 & 0.4 & 0.4 & 0.40 \\
\hline$\beta_{51}$ & 0.41 & $0.41(1)$ & 0.41( & 0.4 & 0.4 & 0.4 & 0.4 & 0. & 1) \\
\hline$\beta_{61}$ & & $0.41(1)$ & $0.41(0$. & & 0.41 & & & & \\
\hline $\log \beta_{22}$ & -3.00 & $-3.02(1)$ & $-3.03(0$ & -3.0 & -2 . & -2.8 & -2.8 & -2.9 & -2 \\
\hline$\beta_{32}$ & -0.03 & $-0.01(1)$ & $-0.01(0$ & -0.0 & -0.0 & -0.0 & -0.0 & -0.0 & -0 \\
\hline$\beta_{42}$ & 0 & $0.29(1)$ & $0.28(0$ & & 0.3 & & & 0.3 & \\
\hline$\beta_{52}$ & 0.33 & & $0.29(0$ & & 0.32 & & & 0.3 & \\
\hline & & & 0.1 & & & & & 0.1 & \\
\hline $\log \beta_{33}$ & -0.99 & $-1.02(1)$ & $-1.02(0.76)$ & -1.0 & $-0.90(4.1)$ & -0.90( & -0.90 & $-0.94(14)$ & -0. \\
\hline & 07 & & $0.73(0$. & & & & & & \\
\hline$\beta_{53}$ & 0.6 & $0.63(1)$ & $0.63(0$. & & & 0.68 & 0.68( & $0.66(6-r)$ & $0.64(3.5)$ \\
\hline$\beta_{-}$ & 0.65 & $0.66(1)$ & $0.66(0.84)$ & $0.66(0.85)$ & $0.71(2.5)$ & $0.71(2.5)$ & $0.71(2.4)$ & $0.69(5.7)$ & $0.67(3.4)$ \\
\hline
\end{tabular}

Table 3: FA example: Posterior median estimates for the three factor model with efficiency in parentheses, based on 100 SMC runs 


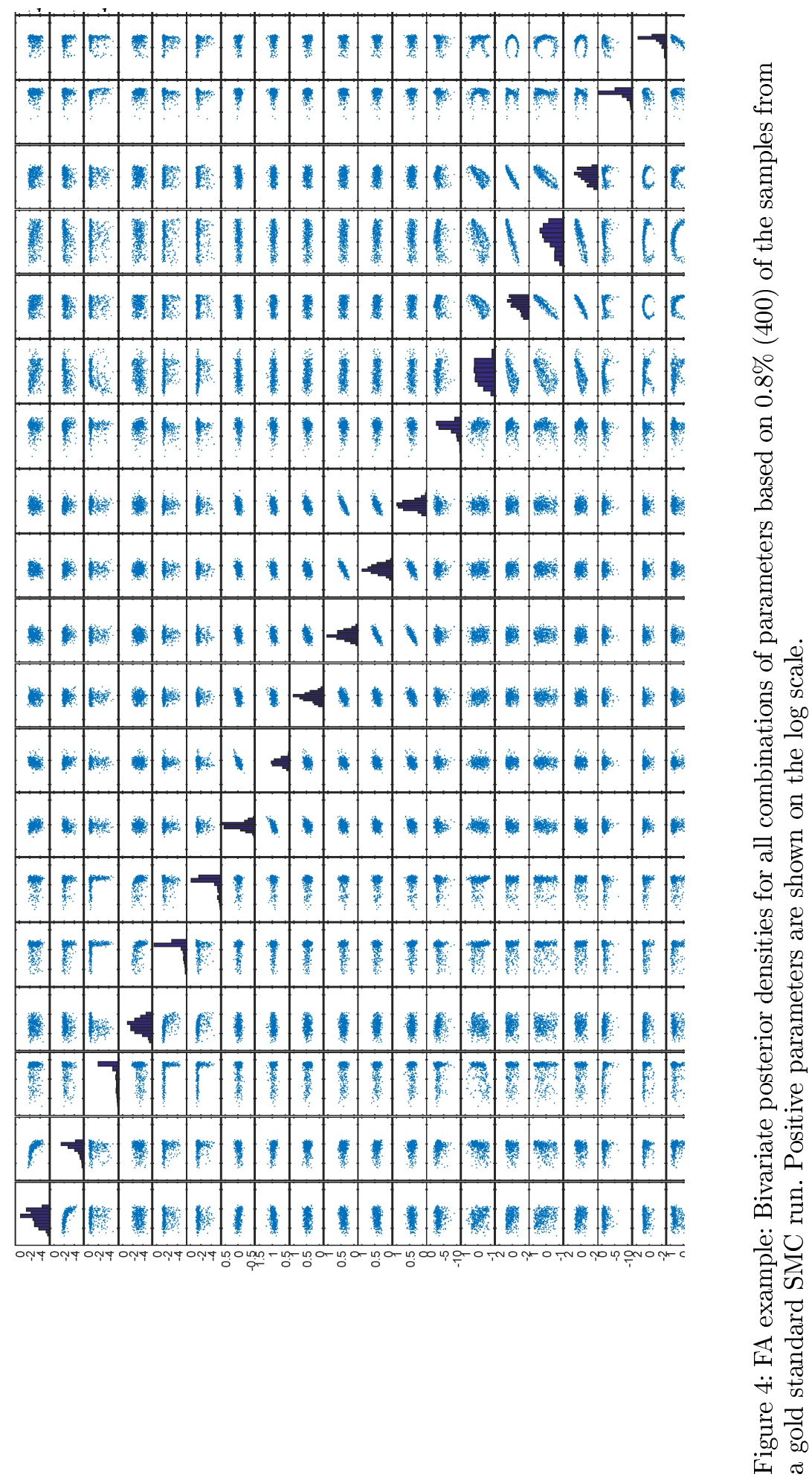




\section{Appendix D: Econometrics Example: Additional Figures}

Posterior estimates of the marginals in this example can be seen in Figure 5. Both the RW and IND kernels recover the marginals well.

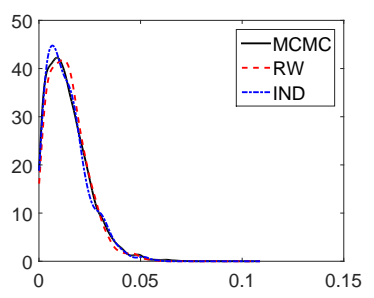

(a) $p_{0}$

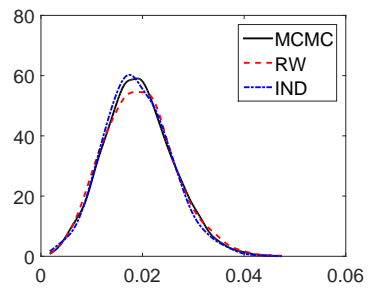

(d) $\rho_{n}$

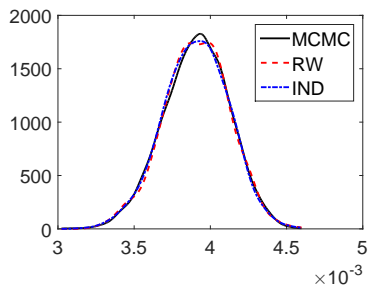

(g) $\phi_{p}^{-}$

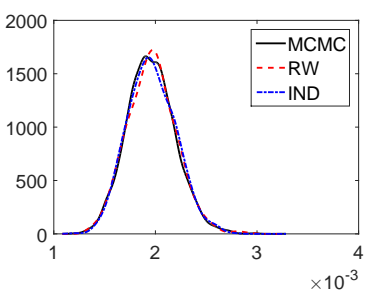

(b) $n_{0}$

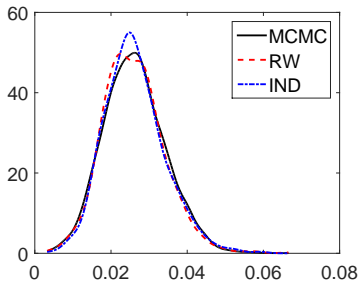

(e) $\phi_{p}^{+}$

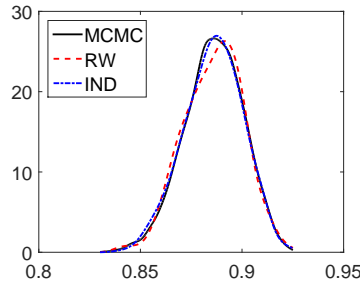

(h) $\phi_{n}^{-}$

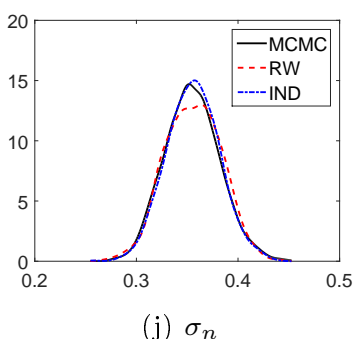

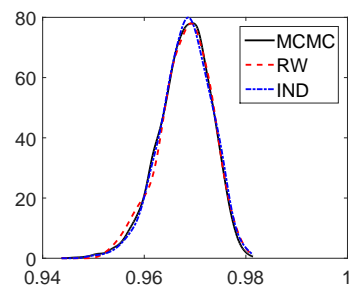

(c) $\rho_{p}$

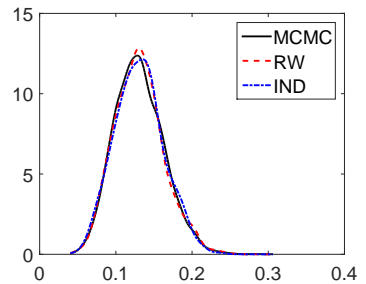

(f) $\phi_{n}^{+}$

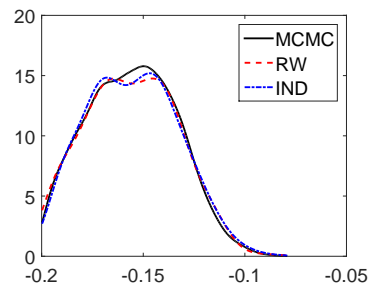

(i) $\sigma_{p}$

Figure 5: BEGE example: Posterior estimates for the BEGE marginals based on a gold standard MCMC run (solid) and single SMC runs with the RW kernel (dash) and IND kernel (dot-dash). Both kernels result in good approximations for the marginals. 


\section{Appendix E: Capture-Recapture Example}

The capture-recapture example used here is based on data collected by Marzolin (1988) on a species of bird called the European Dipper (Cinclus cinclus). Marzolin (1988) collected data based on the capture and recapture of this species over six years. Varying levels of Cormack-Jolly-Seber models (Lebreton et al., 1992) have been applied to the data, but the version used in this analysis is based on a twelve parameter survivor model. Similar analyses based on Bayesian inference for this data set have been performed by others, including Brooks et al. (2000) and Nott et al. (2016).

The parameters for the model are $\phi_{i}$ and $p_{k}$ where $i=1, \ldots, 6$ and $k=2, \ldots, 7$. $\phi_{i}$ represents the probability of survival from year $i$ to year $i+1$ and $p_{k}$ represents the probability of being captured in year $k$.

The likelihood for the model is given below, and based on data $D_{i}$ for the number of birds released in year $i$ and $y_{i k}$ for the number of animals caught in year $k$ out of the number released in year $i$. Here $d_{i}=D_{i}-\sum_{k=i+1}^{7} y_{i k}$ is the number released in year $i$ that are never caught. The corresponding probability of a bird being released in year $i$ and never being caught is $\chi_{i}=1-\sum_{k=i+1}^{7} \phi_{i} p_{k} \prod_{m=i+1}^{k-1} \phi_{m}\left(1-p_{m}\right)$, which is a function of the model parameters. The likelihood is given by

$$
f(\boldsymbol{y} \mid \boldsymbol{\theta}) \propto \prod_{i=1}^{6} \chi_{i}^{d_{i}} \prod_{k=i+1}^{7}\left[\phi_{i} p_{k} \prod_{m=i+1}^{k-1} \phi_{m}\left(1-p_{m}\right)\right]^{y_{i k}},
$$

where $\boldsymbol{\theta}=(\boldsymbol{\phi}, \boldsymbol{p}), \boldsymbol{\phi}=\left(\phi_{1}, \ldots, \phi_{6}\right), \boldsymbol{p}=\left(p_{2}, \ldots, p_{7}\right)$ and $\boldsymbol{y}=\left\{y_{i k}: i=1, \ldots, 6, k=\right.$ $2, \ldots, 7\}$. Due to parameter identifiability issues discussed in Brooks et al. (2000), the parameters $\phi_{6}$ and $p_{7}$ are combined as $\phi_{6} p_{7}$ leading to a total of eleven parameters.

The prior for each component of $\boldsymbol{\theta}$ is set to be $\mathcal{U}(0,1)$, and all components are independent a priori. For the RW proposal, the $j$-th parameter $\boldsymbol{\theta}[j]$ is transformed using $\tilde{\boldsymbol{\theta}}[j]=\log (\boldsymbol{\theta}[j] /(1-\boldsymbol{\theta}[j]))$ for $j=1, \ldots, 11$. The implied prior density for $\tilde{\boldsymbol{\theta}}[j]$ is then $e^{\tilde{\boldsymbol{\theta}}[j]} /\left(1+e^{\tilde{\boldsymbol{\theta}}[j]}\right)^{2}$, for $j=1, \ldots, 11$.

For this example, $100 \mathrm{SMC}$ runs with $N=1,000$ particles are performed. The gold standard for posterior approximation is obtained from a 10 million iteration MCMC run, taking a burn-in of 1,000 iterations and using every 100th sample. The IND proposals are formed using a Gaussian copula model (MGMM copula with one component) with beta marginals since all parameters are probabilities.

\section{Posterior Inference}

Univariate posterior median estimates for different kernels and recycling methods are shown along with their efficiency in Table 4 . The IND proposal consistently outperforms the RW kernel. CIS $S_{P P}$ and DeMix $P$ recycling slightly improve the accuracy for RW and IND, while an even greater improvement can be achieved by recycling all independent proposals. The ESS is 1,000 using only the final $N$ samples and the median ESS

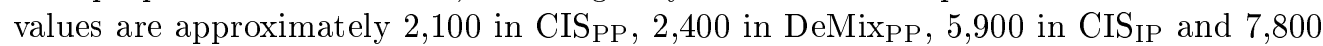


in DeMix IP. Despite the additional computation required in computing the DeMix IP weights, the efficiency appears similar to CIS $_{\text {IP }}$ recycling. The SE results are similar for the 2.5 and 97.5 univariate posterior percentiles (not shown).

\begin{tabular}{|c|c|c|c|c|c|c|c|c|c|}
\hline & \multicolumn{3}{|c|}{ RW } & \multicolumn{5}{|c|}{ IND } \\
\hline & MCMC & SMC & CIS $_{P P}$ & $\operatorname{DeMix}_{\mathrm{PP}}$ & SMC & CIS $_{P P}$ & DeMix $_{P P}$ & CIS $_{I P}$ & DeMix IP \\
\hline$\phi_{1}$ & 0.72 & $0.72(1)$ & $0.72(1.7)$ & $0.72(1.9)$ & $0.72(7.8)$ & $0.72(17)$ & $0.72(15)$ & $0.72(25)$ & $0.72(27)$ \\
\hline$\phi_{2}$ & 0.45 & $0.45(1)$ & $0.45(1.2)$ & $0.45(1.4)$ & $45(5.2)$ & $0.45(11)$ & $0.45(14)$ & $0.45(100)$ & $0.45(190)$ \\
\hline$\phi_{3}$ & 0.48 & $0.48(1)$ & $0.48(1.2)$ & $0.48(1$ & $18(4.6)$ & $0.48(12)$ & $0.48(12)$ & $0.48(67)$ & $0.48(94)$ \\
\hline$\phi_{4}$ & 0.63 & $0.63(1)$ & $0.63(1.7)$ & $0.63(1.9)$ & $0.63(4.6)$ & $0.63(11)$ & $0.63(15)$ & $0.63(18)$ & $0.63(42)$ \\
\hline$\phi_{5}$ & 0.60 & $0.60(1)$ & $0.60(1.8)$ & $0.60(1.8)$ & $0.60(6.4)$ & $0.60(12)$ & $0.60(14)$ & $0.60(66)$ & $0.60(86)$ \\
\hline$p_{2}$ & 0.67 & $0.67(1)$ & $0.67(1.2)$ & $0.67(1.4)$ & $0.67(8.5)$ & $0.67(14)$ & $0.67(16)$ & $0.67(86)$ & $0.67(170)$ \\
\hline$p_{3}$ & 0.88 & $0.88(1)$ & $0.88(1.5)$ & $0.88(1.7)$ & $0.88(9.6)$ & $0.88(23)$ & $0.88(22)$ & $0.88(120)$ & $0.88(79)$ \\
\hline$p_{4}$ & 0.89 & $0.89(1)$ & $0.89(1.0)$ & $0.89(1.1)$ & $0.89(4.8)$ & $0.89(9.3)$ & $0.89(9.8)$ & $0.89(23)$ & $0.89(29)$ \\
\hline$p_{5}$ & 0.88 & $0.88(1)$ & $0.88(1.4)$ & $0.88(1.5)$ & $0.88(5.7)$ & $0.88(15)$ & $0.88(17)$ & $0.88(27)$ & $0.88(44)$ \\
\hline$p_{6}$ & 0.91 & $0.91(1)$ & $0.91(1.8)$ & $0.91(2.2)$ & $0.91(6.7)$ & $0.91(12)$ & $0.91(13)$ & $0.91(47)$ & $0.91(19)$ \\
\hline$\phi_{6} p_{7}$ & 0.53 & $0.53(1)$ & $0.53(1.4)$ & $0.53(1.4)$ & $0.53(6.9)$ & $0.53(13)$ & $0.53(16)$ & $0.53(56)$ & $0.53(80)$ \\
\hline
\end{tabular}

Table 4: Recapture example: Posterior median estimates and efficiency in parentheses, based on 100 SMC runs

\section{Evidence}

Figure 6 shows boxplots of the SMC and IS-based estimates of the log evidence based on 100 runs. IND seems to result in the more efficient standard SMC estimator of the evidence, and the improvement from this best standard SMC estimator to the CIS IP and DeMix ${ }_{I P}$ estimators (Figure 6) is remarkable.

Efficiency of the log evidence estimators can be compared using Table 5. The SMC IND estimator offers a significant improvement over the SMC RW estimator and the best results by far are obtained with the IP evidence estimator. The DeMix IP estimator outperforms the CIS $_{\text {IP }}$ estimator at the cost of additional proposal density computations.

\begin{tabular}{cc|ccc} 
Method & Sampler & $\log \widehat{Z}+348$ & avg. evals & efficiency \\
\hline RW & SMC & -0.14 & $1.7 \times 10^{5}$ & 1.0 \\
& CIS $_{\text {PP }}$ & -0.14 & $"$ & 0.98 \\
& DeMixPP & -0.14 & $"$ & 1.0 \\
IND & SMC & -0.18 & $3.5 \times 10^{4}$ & 18 \\
& CISPP $_{\text {PP }}$ & -0.18 & $"$ & 18 \\
& DeMixPP & -0.18 & $"$ & 18 \\
& CIS IP $_{\text {IP }}$ & -0.22 & $"$ & 4,200 \\
& DeMix $_{\text {IP }}$ & -0.22 & $"$ & 9,800 \\
\hline
\end{tabular}

Table 5: Recapture example: Log mean of the estimated evidence and efficiency. Efficiency is based on VAR rather than MSE.

\section{Appendix F: Ricker Example}

The Ricker model used here is a state space model for the evolution of a population over time (Wood, 2010; Fasiolo et al., 2016) based on the noisily observed Ricker map 


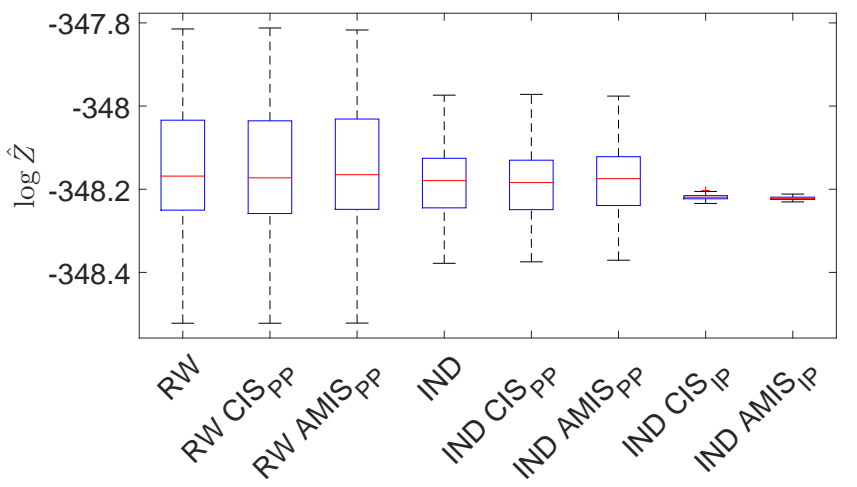

Figure 6: Recapture example: Boxplots of $100 \log$ evidence estimates for all combinations of recycling method and MCMC kernel.

(Turchin, 2003). The population $N_{t}$ is modelled at time $t$ under the discrete time stochastic process

$$
N_{t+1}=r N_{t} e^{-N_{t}+z_{t+1}},
$$

where $z_{t} \sim \mathcal{N}\left(0, \sigma^{2}\right)$ and we set $N_{0}=1$. The true population count $N_{t}$ is observed with error. We denote the corresponding observation $y_{t}$ and assume $y_{t} \sim \operatorname{Pois}\left(\phi N_{t}\right)$. This creates a state space model. The parameters $\boldsymbol{\theta}=(\log r, \log \phi, \log \sigma)$ are the natural logarithm of the population growth rate, of the scale parameter and of the standard deviation of the environmental noise, respectively. Exact-approximate methods, specifically particle MCMC (Andrieu et al., 2010) and the SMC method of Duan and Fulop (2015), are used here since an unbiased estimate of the intractable likelihood, $f\left(\mathbf{y}_{1: t} \mid \boldsymbol{\theta}\right)$ where $\boldsymbol{y}_{1: t}$ denotes the data up to the current $t$, is available. We use the standard bootstrap particle filter (Gordon et al., 1993) with $J=1000$ particles. It is difficult to perform inference on this sort of non-linear dynamic model due to irregularities in some parts of the parameter space. More details on the specific challenges that this model presents for standard MCMC methods can be found in Wood (2010) and Fasiolo et al. (2016). The benefit of using an independent copula proposal in this example is that it is derivative-free so it can easily be applied even when the exact likelihood is unavailable as is the case here.

The IND proposals are formed using a MGMM copula model with two components. Given the following uniform priors

$$
\begin{aligned}
\log r & \sim \mathcal{U}(2,5) \\
\log \phi & \sim \mathcal{U}(1.61,3) \\
\log \sigma & \sim \mathcal{U}(-3,-0.22)
\end{aligned}
$$

it is appropriate to use beta marginals for the independent proposals after transforming the parameters to $[0,1]$. The gold standard for this example is based on 1 million 
iterations of particle MCMC (with a burn-in of 1,000 iterations and taking every 100th sample) and 100 SMC runs with $N=1000$ are performed for the RW and IND kernels.

\section{Posterior Inference}

Posterior median estimates and their relative efficiency can be seen in Table 6 for this example. Consistently good results can be achieved by recycling all independent proposals. The ESS for CIS IP recycling is between 5300 and 7500 and for DeMix ${ }_{I P}$ recycling is between 5800 and 8400 . The ESS values from recycling all proposals are substantially higher than the ESS from CIS PP recycling (2000-2500) and DeMix $_{P P}$ (2300-2700) recycling. The IND proposals are more efficient than the RW kernel both before and after recycling. The results are similar for the $2.5 \%$ and $97.5 \%$ quantiles.

\begin{tabular}{|c|c|c|c|c|c|c|c|c|c|}
\hline & $\begin{array}{r}\text { GOLD } \\
\text { MCMC }\end{array}$ & $\mathrm{SMC}$ & $\begin{array}{l}\mathrm{RW} \\
\mathrm{CIS}_{\mathrm{PP}}\end{array}$ & $\operatorname{DeMix}_{P P}$ & SMC & $\mathrm{CIS}_{\mathrm{PP}}$ & $\begin{array}{c}\text { IND } \\
\text { DeMixpP }\end{array}$ & CIS $_{I P}$ & DeMix $_{\text {IP }}$ \\
\hline $\log r$ & 3.82 & $3.82(1)$ & $3.82(2.7)$ & $3.82(3.1)$ & $3.82(4.8)$ & $3.82(10)$ & $3.82(12)$ & $3.82(81)$ & $3.82(85)$ \\
\hline $\log \phi$ & 2.34 & $2.34(1)$ & $2.34(2.7)$ & $2.34(3.2)$ & $2.34(3.4)$ & $2.34(6.9)$ & $2.34(7.4)$ & $2.34(31)$ & $2.34(25)$ \\
\hline $\log \sigma$ & -0.96 & $-0.96(1)$ & $-0.96(2.1)$ & $-0.96(2.4)$ & $-0.96(3.7)$ & $-0.96(5.8)$ & $-0.96(6.9)$ & $-0.96(13)$ & $-0.96(16)$ \\
\hline
\end{tabular}

Table 6: Ricker example: Posterior median estimates and efficiency in parentheses, based on 100 SMC runs

\section{Evidence}

The SMC and IS-based estimators of the evidence are shown via boxplots in Figure 7 and Table 7 displays the efficiency of each method. The independent proposals are more efficient in estimating the evidence and a remarkable improvement is achieved by recycling all independent proposals to estimate the evidence.

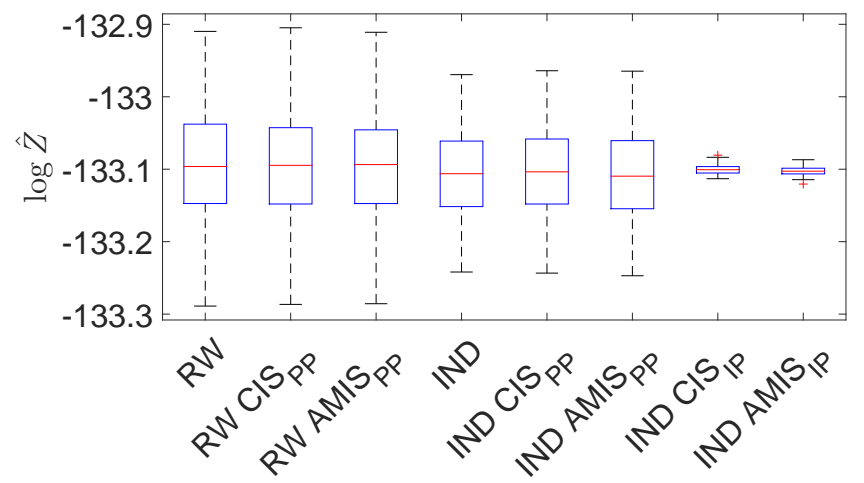

Figure 7: Ricker example: Boxplots of $100 \log$ evidence estimates for all combinations of recycling method and MCMC kernel. 


\begin{tabular}{cc|ccc} 
Method & Sampler & $\log \widehat{Z}+133$ & avg. evals & efficiency \\
\hline RW & SMC & -0.09 & $8.7 \times 10^{4}$ & 1.0 \\
& CIS $_{P P}$ & -0.09 & $"$ & 0.99 \\
& DeMix & -0.09 & $"$ & 1.1 \\
IND & SMC & -0.10 & $2.4 \times 10^{4}$ & 5.3 \\
& CIS $_{P P}$ & -0.10 & $"$ & 5.1 \\
& DeMixPP & -0.11 & $"$ & 5.4 \\
& CIS IP $_{\text {IP }}$ & -0.10 & $"$ & 440 \\
& DeMix $_{\text {IP }}$ & -0.10 & $"$ & 560 \\
\hline
\end{tabular}

Table 7: Ricker example: Log mean of the estimated evidence and efficiency. Efficiency is based on VAR rather than MSE.

\section{Appendix G: ODE Model for Biochemical Pathways}

With the exception of the three factor model in Section 4.1 of the main paper, the use of independent proposals resulted in a significant reduction in the uncertainty of estimates of posterior expectations and the evidence. We found that the posterior distributions in these examples were quite regular and could be modelled well with our copula model. The purpose of this example is to consider another posterior distribution with significant irregularities that will motivate further research on this topic to increase the capabilities of SMC with an independent proposal.

A nonlinear ordinary differential equation (ODE) system for modelling biochemical pathways is investigated here through SMC. This ten-dimensional example is based on the Michaelis-Menten kinetic law for defining the activation of protein $R$ and is used for investigating the enzymatic activation of protein $R$ into its active form Rpp by enzyme $S$.

The four-dimensional system of coupled ODEs shown below has been described in Geyer (1991) and investigated from a Bayesian context in Girolami (2008):

$$
\begin{gathered}
\frac{d S}{d t}=-k_{1} S \\
\frac{d D}{d t}=k_{1} S \\
\frac{d R}{d t}=-\frac{V_{1} R S}{K m_{1}+R}+\frac{V_{2} R p p}{K m_{2}+R p p} \\
\frac{d R p p}{d t}=\frac{V_{1} R S}{K m_{1}+R}-\frac{V_{2} R p p}{K m_{2}+R p p} .
\end{gathered}
$$

According to this system, the total of $R$ and $R p p$ is constant and the total of $S$ and $D$ is constant.

The first five parameters for this application are given by $k_{1}, V_{1}, K m_{1}, V_{2}$ and $K m_{2}$ and the next four are given by the initial values for the variables, $S_{0}, D_{0}, R_{0}$ and $R p p_{0}$. Here we generate data from the model and collect data at 20 distinct time points based on the following parameter configuration specified by Girolami (2008):

$$
\begin{gathered}
{\left[\begin{array}{ccccccccc}
k_{1} & V_{1} & K m_{1} & K m_{2} & V_{2} & S_{0} & D_{0} & R_{0} & R p p_{0}
\end{array}\right]=} \\
{\left[\begin{array}{lllllllll}
0.05 & 0.2 & 0.1 & 0.1 & 0.1 & 1 & 0 & 1 & 0
\end{array}\right] .}
\end{gathered}
$$


We assume that only $R p p$ can be observed and that it is observed with error. We denote the corresponding observations $y$ and following Girolami (2008), we assume $y(t) \sim \mathcal{N}\left(R p p(t), \sigma^{2}\right)$ where $\sigma$ is chosen to be 0.02 .

A plot of the true levels of $R p p$ based on the first 9 parameters along with the observed Rpp using $\sigma=0.02$ can be seen in Figure 8. The time period started at zero and observations are made at every three units of time (up to 57) as this is where the level of $R p p$ begins to asymptote to zero. The data is denoted as $\boldsymbol{y}=\{y(t): t=0,3, \ldots, 57\}$.

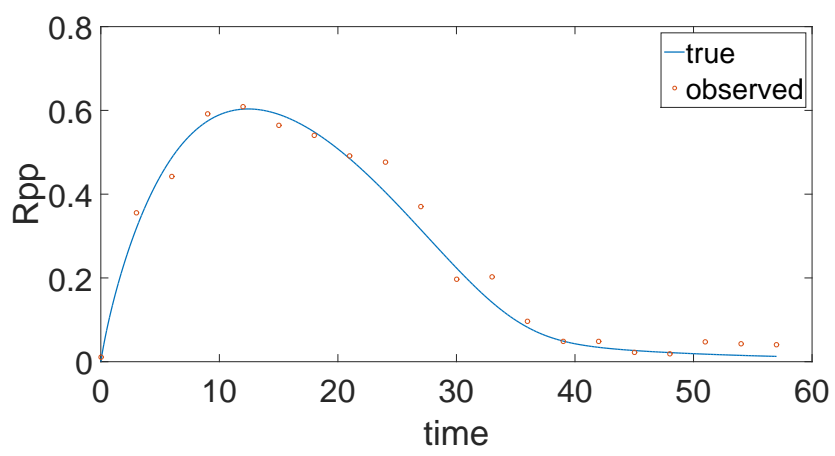

Figure 8: ODE example: True and observed Rpp levels.

Girolami (2008) gives the covariance structure of the model parameters, suggesting that a number of the variables are highly correlated. A closer investigation reveals several practical and structural identifiability issues in this ODE model (see Raue et al. (2009) for a review of identifiability analysis). Since only $R p p$ is observed, no information is obtained about the initial value of $D$, making $D_{0}$ practically non-identifiable. There is also a number of issues caused by structural identifiability resulting from the form of the model. Attempting to estimate the standard deviation of the Gaussian noise causes further identifiability issues. The Gamma priors below of the form $\mathcal{G}(\alpha, \beta)$ with mean $\alpha \beta$ and variance $\alpha \beta^{2}$ do help to enforce some weak identifiability,

$$
\begin{gathered}
k_{1}, V_{1}, K m_{1}, V_{2}, K m_{2}, \sigma \sim \mathcal{G}(1,1) \\
S_{0}, R_{0} \sim \mathcal{G}(5,0.2) \\
D_{0}, R p p_{0} \sim \mathcal{G}(1,0.1) .
\end{gathered}
$$

However the problem remains challenging even without estimating $\sigma$. For the purposes of this paper, analysis is performed on the already challenging 9 parameter example with $\boldsymbol{\theta}=\left(\log k_{1}, \log V_{1}, \log K m_{1}, \log K m_{2}, \log V_{2}, \log S_{0}, \log D_{0}, \log R_{0}, \log R p p_{0}\right)$ and $\sigma=0.02$. The posterior for this 9 parameter example is so complex that when fitting a MGMM to the posterior samples of a long MCMC run, the BIC was still decreasing even after fitting 15 components.

To model each of the marginals, five component GMMs are used. Taking advantage of the fact that no information is obtained about $D_{0}$, this parameter is updated 
independently of the other parameters using its fitted marginal distribution. We also update $R p p_{0}$ independently since it is well identified because a noisy observation of the $R p p$ variable is taken at $t=0$. Due to the complexities of the dependence structure, proposals for the remaining seven parameters are based on a MGMM copula model with six components.

One hundred SMC runs with $N=2000$ particles in each are performed for this example. The gold standard posterior approximation is based on an MCMC run with 10 million iterations, with a 100,000 iteration burn-in and thinning by taking every 1000th sample. The bivariate posterior density estimates from the MCMC run are shown in Figure 9. The combinations of $k_{1}$ and $V_{2}, K m_{2}$ and $V_{2}$, and to a lesser extent $V_{1}$ and $S_{0}$ appear to have the most complex dependencies. 


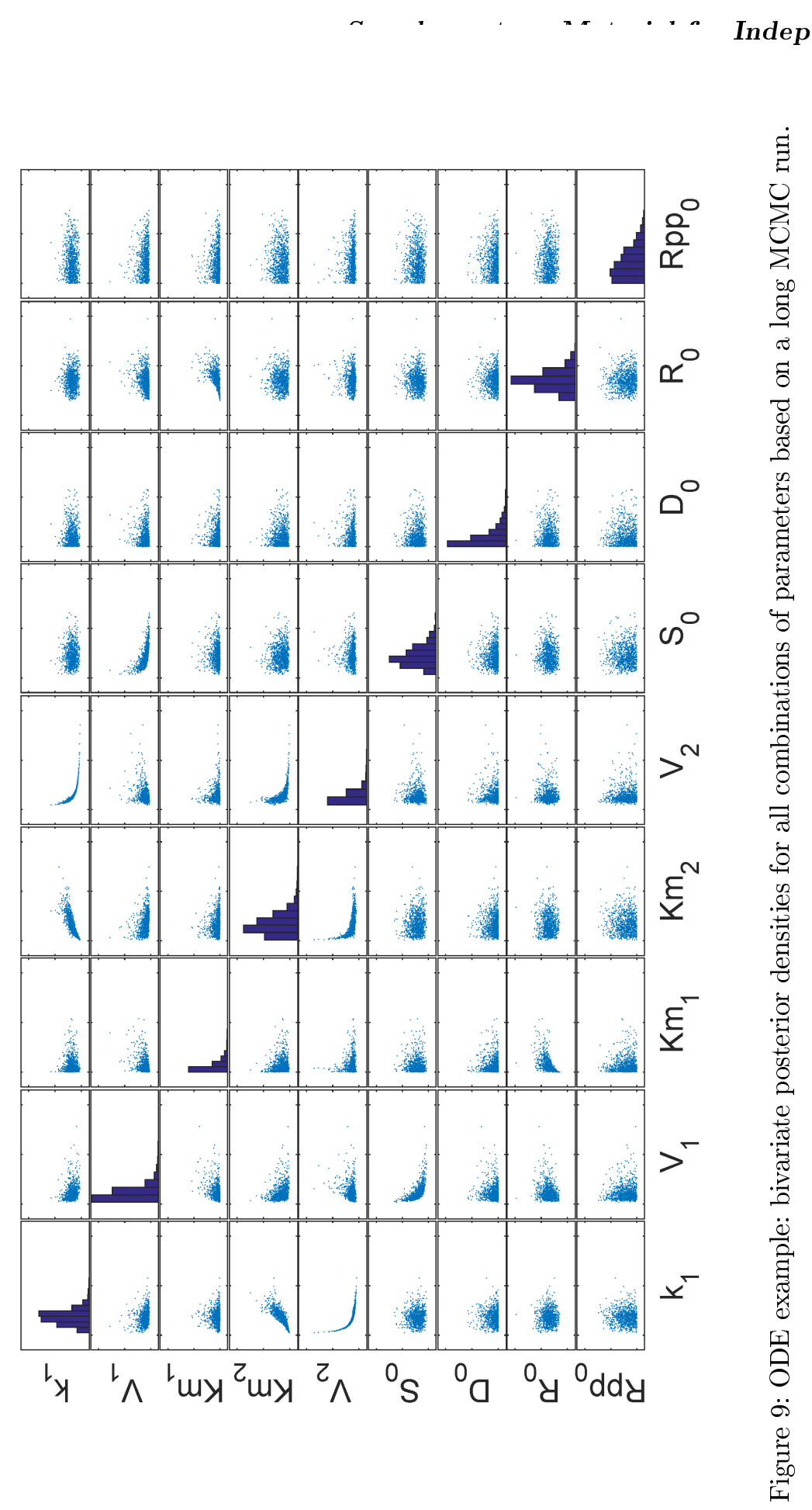




\section{Posterior Inference}

Given the complexities of the posterior, covering the tails of the target is challenging in this application. Figure 10 shows posterior estimates for the marginals based on the gold standard long MCMC run. Also shown in Figure 10 are results based on a single SMC run using the RW and IND proposals. From inspection, it appears that RW covers the tails of the target whereas there are issues with tail coverage in IND for parameters $k_{1}, K m_{2}$ and $V_{2}$.

Although a long MCMC run and SMC RW are available for comparison here, these methods are expensive to perform and it is not always feasible to perform such long runs. Using the RW kernel with 2000 particles in SMC requires an average of 3.6 million log-likelihood computations, while IND requires an average of approximately 600,000 log-likelihood computations. Other kernels that were tried for this example, including the Liu-West proposal, also failed to cover the tails of the target (results not shown).

One advantage we found with the IP recycling is that the ESS was often small (substantially less than $N$ ), caused by particles in the tails being assigned high weights. Thus in some cases lack of tail coverage can be diagnosed. Unfortunately, though, we found that the ESS can be misleadingly high even when tail coverage is an issue.

Shown in Tables 8, 9 and 10 are posterior median, $2.5 \%$ quantile and $97.5 \%$ quantile estimates and their related efficiency values. The concerning parts of the posterior approximation, the lower tails for $k_{1}$ and $K m_{2}$ and the upper tail for $V_{2}$, are improved by recycling all independent proposals. However, the extreme weights associated with proposals in the tails of $k_{1}, K m_{2}$ and $V_{2}$ have a detrimental effect on the efficiency of posterior estimates for other parameters. CIS $\mathrm{PP}_{\mathrm{P}}$ and DeMix $\mathrm{PP}_{\mathrm{P}}$ recycling have performed poorly in general for both the RW and IND proposals, and the estimated posterior based on this weighting scheme has issues with tail coverage.

The improvements from IP recycling suggest that reusing past particles can help to improve the accuracy of posterior estimates. The small ESS after recycling remains an issue for using the weighted samples in posterior inference, but can also be considered an advantage as it brings attention to the issues with tail coverage in the original samples. This challenging application motivates further investigation into efficient independent proposals which also cover the tails of the target.

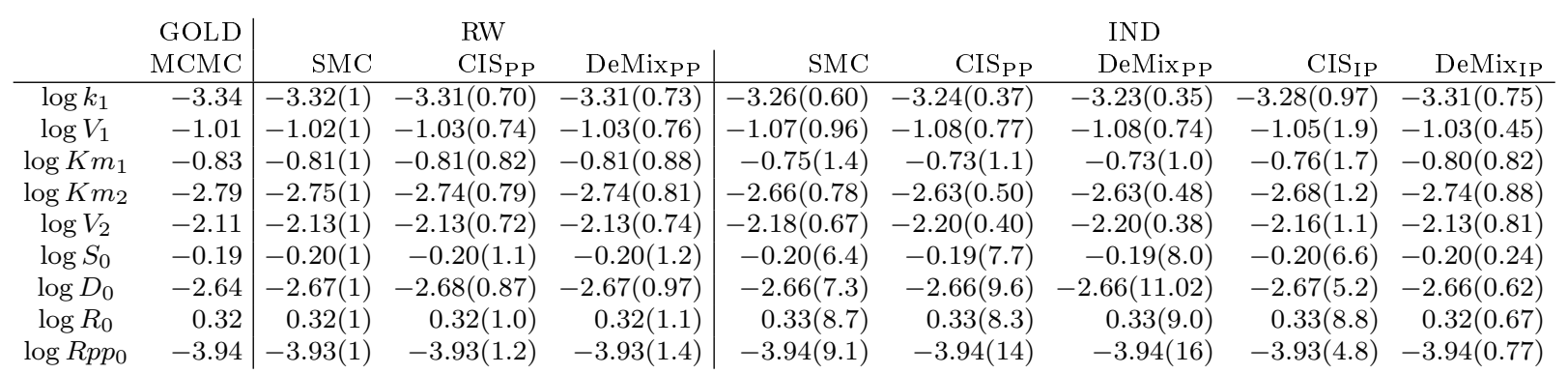

Table 8: ODE example: Posterior median estimates and efficiency in parentheses, based on 100 SMC runs 


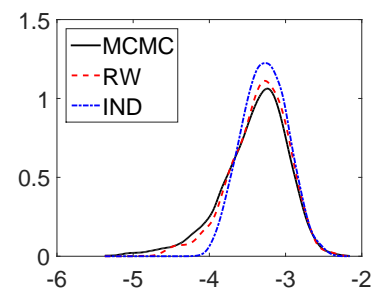

(a) $\log k_{1}$

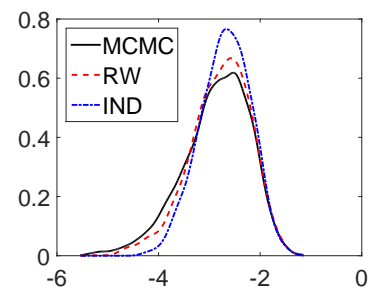

(d) $\log K m_{2}$

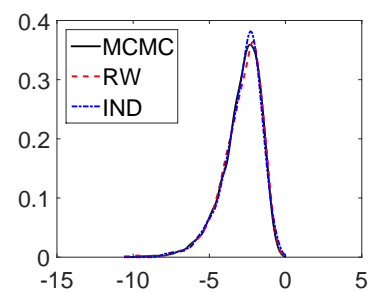

(g) $\log D_{0}$

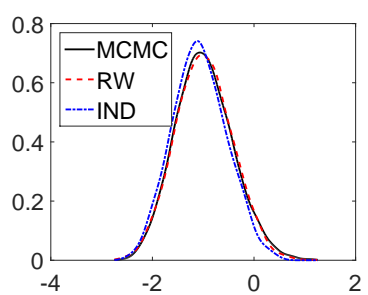

(b) $\log V_{1}$

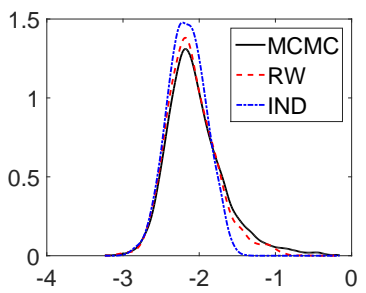

(e) $\log V_{2}$

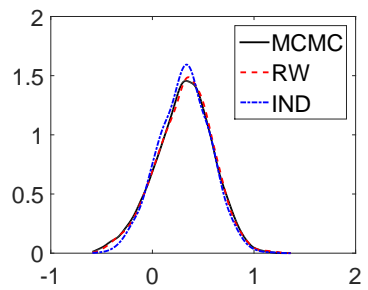

(h) $\log R_{0}$

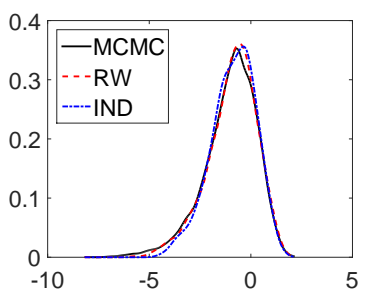

(c) $\log K m_{1}$

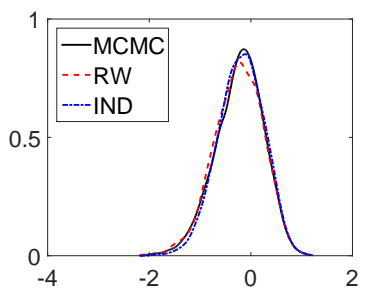

(f) $\log S_{0}$

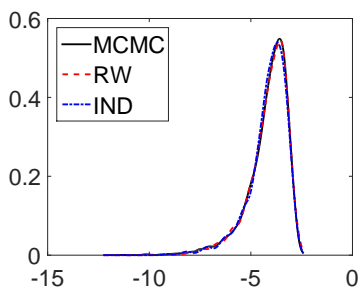

(i) $\log R p p_{0}$

Figure 10: ODE example: Posterior estimates for the ODE example parameters (on log scale) based on a gold standard MCMC run (solid) and single SMC runs with the RW kernel (dash) and IND kernel (dot-dash).

\begin{tabular}{cr|rrr|rrrrr}
\multicolumn{9}{c}{ GOLD } & \multicolumn{9}{c|}{ RW } & \multicolumn{3}{c}{ IND } \\
& MCMC & SMC & CIS PP & DeMix & SMC & CIS $_{\text {PP }}$ & DeMix $_{\text {PP }}$ & CIS $_{\text {IP }}$ & DeMix $_{\text {IP }}$ \\
\hline $\log k_{1}$ & -4.46 & $-4.20(1)$ & $-4.18(0.84)$ & $-4.18(0.84)$ & $-3.83(1.1)$ & $-3.78(0.95)$ & $-3.78(0.95)$ & $-3.91(1.5)$ & $-3.97(1.8)$ \\
$\log V_{1}$ & -2.02 & $-2.03(1)$ & $-2.03(2.0)$ & $-2.03(1.9)$ & $-2.04(4.5)$ & $-2.04(6.8)$ & $-2.04(6.4)$ & $-2.04(4.9)$ & $-2.03(2.8)$ \\
$\log K m_{1}$ & -4.12 & $-3.76(1)$ & $-3.75(0.98)$ & $-3.76(1.0)$ & $-3.34(1.9)$ & $-3.27(1.6)$ & $-3.24(1.5)$ & $-3.52(2.7)$ & $-3.55(2.7)$ \\
$\log K m_{2}$ & -4.38 & $-4.08(1)$ & $-4.06(0.84)$ & $-4.06(0.82)$ & $-3.64(1.1)$ & $-3.58(0.95)$ & $-3.58(0.93)$ & $-3.76(1.5)$ & $-3.84(1.9)$ \\
$\log V_{2}$ & -2.64 & $-2.65(1)$ & $-2.65(2.3)$ & $-2.65(3.7)$ & $-2.65(5.3)$ & $-2.67(2.6)$ & $-2.68(1.6)$ & $-2.66(2.3)$ & $-2.65(8.5)$ \\
$\log S_{0}$ & -1.24 & $-1.23(1)$ & $-1.23(1.6)$ & $-1.22(1.7)$ & $-1.18(1.9)$ & $-1.17(1.6)$ & $-1.17(1.6)$ & $-1.22(2.5)$ & $-1.22(1.8)$ \\
$\log D_{0}$ & -6.05 & $-5.95(1)$ & $-5.96(1.2)$ & $-5.97(1.2)$ & $-5.91(5.9)$ & $-5.90(5.8)$ & $-5.90(5.9)$ & $-5.93(5.4)$ & $-5.91(3.3)$ \\
$\log R_{0}$ & -0.28 & $-0.26(1)$ & $-0.26(0.85)$ & $-0.26(0.92)$ & $-0.21(1.5)$ & $-0.20(1.2)$ & $-0.20(1.1)$ & $-0.23(1.9)$ & $-0.23(1.6)$ \\
$\log R p p_{0}$ & -6.89 & $-6.74(1)$ & $-6.73(1.0)$ & $-6.74(1.1)$ & $-6.75(7.6)$ & $-6.72(7.5)$ & $-6.72(7.9)$ & $-6.74(6.4)$ & $-6.72(5.2)$
\end{tabular}

Table 9: ODE example: Posterior lower $2.5 \%$ quantile estimates and efficiency in parentheses, based on 100 SMC runs 


\begin{tabular}{cr|rrr|rrrrr}
\multicolumn{9}{c|}{ GOLD } & \multicolumn{9}{c}{ RW } & \multicolumn{3}{c}{ IND } \\
& MCMC & SMC & CISPP & DeMix PP & SMC & CISPP & DeMix & CIS $_{\text {IP }}$ & DeMix $_{\text {IP }}$ \\
\hline $\log k_{1}$ & -2.71 & $-2.71(1)$ & $-2.71(2.6)$ & $-2.71(3.9)$ & $-2.71(7.9)$ & $-2.69(3.5)$ & $-2.68(2.3)$ & $-2.68(1.1)$ & $-2.71(10)$ \\
$\log V_{1}$ & 0.19 & $0.13(1)$ & $0.12(0.91)$ & $0.12(0.91)$ & $0.04(1.3)$ & $0.02(1.1)$ & $0.02(1.1)$ & $0.10(2.1)$ & $0.10(1.6)$ \\
$\log K m_{1}$ & 1.04 & $1.00(1)$ & $1.00(1.0)$ & $1.00(1.1)$ & $1.00(11)$ & $1.01(14)$ & $1.01(13)$ & $1.03(10)$ & $1.01(7.5)$ \\
$\log K m_{2}$ & -1.79 & $-1.81(1)$ & $-1.80(2.5)$ & $-1.80(2.2)$ & $-1.79(11)$ & $-1.77(8.3)$ & $-1.77(6.9)$ & $-1.76(0.61)$ & $-1.80(4.8)$ \\
$\log V_{2}$ & -1.07 & $-1.33(1)$ & $-1.35(0.86)$ & $-1.35(0.87)$ & $-1.69(1.2)$ & $-1.73(1.1)$ & $-1.73(1.1)$ & $-1.60(1.6)$ & $-1.55(2.0)$ \\
$\log S_{0}$ & 0.61 & $0.61(1)$ & $0.61(1.7)$ & $0.61(1.8)$ & $0.61(4.4)$ & $0.60(5.0)$ & $0.60(5.9)$ & $0.61(3.0)$ & $0.61(2.0)$ \\
$\log D_{0}$ & -1.02 & $-1.00(1)$ & $-1.00(1.4)$ & $-1.00(1.8)$ & $-1.01(6.1)$ & $-0.99(6.1)$ & $-0.99(6.1)$ & $-0.99(3.4)$ & $-1.01(5.7)$ \\
$\log R_{0}$ & 0.83 & $0.82(1)$ & $0.82(1.4)$ & $0.82(1.4)$ & $0.81(3.4)$ & $0.81(3.6)$ & $0.81(3.5)$ & $0.82(3.6)$ & $0.82(3.1)$ \\
$\log R p p_{0}$ & -2.90 & $-2.92(1)$ & $-2.92(1.4)$ & $-2.92(1.1)$ & $-2.93(2.3)$ & $-2.93(4.0)$ & $-2.93(3.2)$ & $-2.92(5.9)$ & $-2.93(2.5)$
\end{tabular}

Table 10: ODE example: Posterior upper $97.5 \%$ quantile estimates and efficiency in parentheses, based on $100 \mathrm{SMC}$ runs

\section{Evidence}

Boxplots of the log evidence estimates based on 100 SMC runs with 2000 particles can be seen in Figure 11. There is no guarantee that these estimators of the evidence are unbiased since adaptive methods have been used, and this is noticeable in Figure 11 where at least one of the estimators is clearly biased. The IP estimators of the evidence are closer to the SMC RW estimator of the evidence than the SMC IND estimator. It is interesting to observe that relatively precise estimates of the evidence can be obtained with CIS $_{\text {IP }}$ despite extreme weights and a proposal which may not fully cover the tails. The outlying $\log$ evidence estimates of 22.59 and 22.48 for DeMix IP are associated with the two lowest ESS values of 5.2 and 7.0, respectively.

Efficiency results are shown in Table 11, although we note that there is no gold standard of evidence estimation so this measure is based on variance and does not

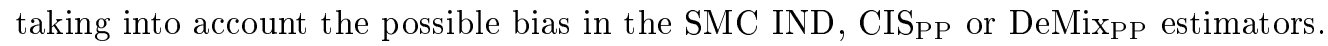
The IP evidence estimators are more efficient by this measure than the SMC RW and $\mathrm{PP}$ recycling estimators. The DeMix $\mathrm{IP}_{\mathrm{P}}$ weighting scheme does not offer any advantage over the CIS $\mathrm{CP}_{\mathrm{IP}}$ scheme in this example. The deterministic multiple mixture weights in DeMix generally aid in avoiding extreme weights, but if an extreme weight does occur it can significantly reduce the overall ESS. On the other hand, the effect of extreme weights in CIS recycling is limited to affecting the component of the ESS from that temperature. As a result, extreme weights for proposals from early temperatures tend to have a smaller effect on the ESS and overall performance of the CIS recycling method.

\begin{tabular}{cc|ccc} 
Method & Sampler & $\log \widehat{Z}-21$ & avg. evals & efficiency \\
\hline RW & SMC & 0.97 & $4.8 \times 10^{5}$ & 1.0 \\
& CIS $_{P P}$ & 0.96 & $" 1$ & 1.0 \\
& DeMix $_{P P}$ & 0.97 & $"$ & 1.0 \\
IND & SMC $_{4}$ & 0.18 & $1.3 \times 10^{5}$ & 17 \\
& CIS $_{P P}$ & 0.17 & $"$ & 17 \\
& DeMix $_{P P}$ & 0.18 & $"$ & 17 \\
& CIS $_{\text {IP }}$ & 0.92 & $"$ & 66 \\
& DeMix $_{\text {IP }}$ & 0.98 & $"$ & 10 \\
\hline
\end{tabular}

Table 11: ODE example: Log mean of the estimated evidence and efficiency. Efficiency is based on VAR rather than MSE. 


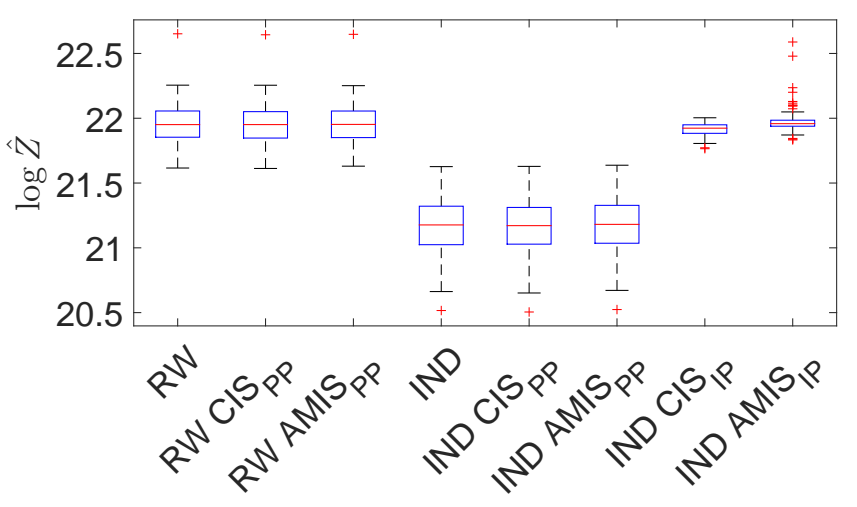

Figure 11: ODE example: Boxplots of $100 \log$ evidence estimates for all combinations of recycling method and MCMC kernel.

\section{Appendix $\mathrm{H}$ : Alternative Implementation Without $\mathrm{MH}$ Correction}

In the independent SMC method described in the main paper, the weighted particle set $\left\{W_{t}^{i}, \boldsymbol{\theta}_{t-1}^{i}\right\}_{i=1}^{N}$ is resampled and independent proposals from $q^{\boldsymbol{\phi}_{t}}(\cdot)$ are used as proposals in a MH-MCMC step. Rather than resampling and diversifying the previous particles, one could directly use the new particles $\left\{\boldsymbol{\theta}_{t}^{i}\right\}_{i=1}^{N} \sim q^{\phi_{t}}(\cdot)$ by applying the unnormalised IS weights

$$
w_{t}^{i}=\frac{f\left(\mathbf{y} \mid \boldsymbol{\theta}_{t}^{i}\right)^{\gamma_{t}} \pi\left(\boldsymbol{\theta}_{t}^{i}\right)}{q^{\phi_{t}}\left(\boldsymbol{\theta}_{t}^{i}\right)},
$$

for $i=1, \ldots, N$ so that the weighted particle set targets $\pi_{t}$. This alternative method is described here and shown in Algorithm 1.

In Algorithm 1, the particle set representing $\pi_{t}$ at the end of line 14 is weighted and this affects both the choice of the temperature schedule and the quality of the (power) posterior approximations. For unweighted particle sets, the discrepancy between $\pi_{t-1}$ and $\pi_{t}$ can be held constant by choosing the temperatures to achieve a fixed ESS. Given that the particle sets are weighted in this alternative algorithm, the discrepancy between distributions can be kept constant by maintaining a fixed conditional ESS (CESS, Zhou et al. (2016)),

$$
\operatorname{CESS}_{t}=\frac{N\left(\sum_{i=1}^{N} W_{t-1}^{i} f\left(\mathbf{y} \mid \boldsymbol{\theta}_{t-1}^{i}\right)^{\gamma_{t}-\gamma_{t-1}}\right)^{2}}{\sum_{i=1}^{N} W_{t-1}^{i}\left(f\left(\mathbf{y} \mid \boldsymbol{\theta}_{t-1}^{i}\right)^{\gamma_{t}-\gamma_{t-1}}\right)^{2}} .
$$

The CESS makes it feasible to choose the temperature schedule under these conditions, but there is a risk that the weights will continue to degenerate and lead to increasingly worse importance distributions.

One may attempt to reduce the weight degeneracy by continuing to draw proposals

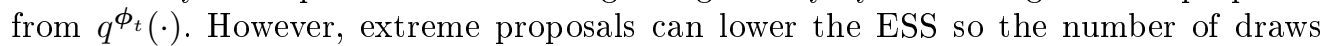


Input : The number of particles for each of temperatures $\left\{N_{t}\right\}_{t=0}^{T}$, the prior distribution $\pi(\boldsymbol{\theta})$, a family of proposal distributions $q^{\phi_{t}}$ and the value of $\rho$ for choosing temperatures such that the CESS of particles is maintained at $\rho N$.

Output: SMC samples from each of the $T+1$ power posteriors, $\left\{W_{t}^{i}, \boldsymbol{\theta}_{t}^{i}\right\}_{i=1}^{N}$ for

1 Set $t=0$ $t=0, \ldots, T$ and a logged estimate of the evidence, $\log \widehat{Z}$.

2 Set $\log \widehat{Z}=0$

3 Set $W_{0}^{i}=\frac{1}{N}$ for $i=1, \ldots, N$

4 Simulate $\boldsymbol{\theta}_{0}^{i} \stackrel{i i d}{\sim} \pi(\cdot)$ for $i=1, \ldots, N$

5 while $\gamma_{t} \neq 1$ do

$6 \quad$ Set $t \leftarrow t+1$

$7 \quad$ Compute $\gamma_{t}$ using the bisection method to maintain $\operatorname{CESS}_{t} \approx \rho N$

$8 \quad$ Re-weight $w_{t}^{i}=W_{t-1}^{i} p\left(\boldsymbol{y} \mid \boldsymbol{\theta}_{t-1}^{i}\right)^{\gamma_{t}-\gamma_{t-1}}$ for $i=1, \ldots, N$

$9 \quad$ Compute normalised weights $W_{t}^{i}=\frac{w_{t}^{i}}{\sum_{k=1}^{N} w_{t}^{k}}$ for $i=1, \ldots, N$

10 Compute $\log \widehat{Z} \leftarrow \log \widehat{Z}+\log \sum_{i=1}^{N} w_{i}^{t}$

11 Estimate $\phi_{t}$ from the particle set $\left\{W_{t}^{i}, \boldsymbol{\theta}_{t-1}^{i}\right\}_{i=1}^{N}$

12 Draw $\left\{\boldsymbol{\theta}_{t}^{i}\right\}_{i=1}^{N_{t}} \sim q^{\boldsymbol{\phi}_{t}(\cdot)}$

$13 \quad$ Redefine and compute $w_{t}^{i}=\frac{f\left(\mathbf{y} \mid \boldsymbol{\theta}_{t}^{i}\right)^{\gamma_{t}} \pi\left(\boldsymbol{\theta}_{t}^{i}\right)}{q^{\phi}\left(\boldsymbol{\theta}_{t}^{i}\right)}$

14 Compute normalised weights $W_{t}^{i}=\frac{w_{t}^{i}}{\sum_{k=1}^{N} w_{t}^{k}}$ for $i=1, \ldots, N$

15 end

Algorithm 1: Alternative independent SMC algorithm based on an IS correction at the move step instead of MCMC. 
required to improve the approximation to a given level is unknown a priori. This may be prohibitively expensive, even without considering how using iterative sequences of draws would reduce the ability to use parallel architecture.

This alternative independent SMC method is similar to sequential importance sampling (SIS) and it may be useful in some simple examples where it is relatively easy to find efficient independent proposals. In general we consider this method to be less appealing than a $\mathrm{MH}$ correction because of the difficulty in controlling the ESS.

\section{References}

Andrieu, C., Doucet, A., and Holenstein, R. (2010). "Particle Markov chain Monte Carlo methods." Journal of the Royal Statistical Society: Series B (Statistical Methodology), 72(3): 269-342. 11

Brooks, S. P., Catchpole, E. A., and Morgan, B. J. T. (2000). "Bayesian animal survival estimation." Statistical Sciences, 15(4): 357-376. 9

Duan, J.-C. and Fulop, A. (2015). "Density-tempered marginalised sequential Monte Carlo sampler." Journal of Business and Economics Statistics, 33(2): 192-202. 11

Fasiolo, M., Pya, N., and Wood, S. N. (2016). "A comparison of inferential methods for highly non-linear state space models in ecology and epidemiology." Statistical Science, 31(1): 96-118. 10, 11

Geyer, C. (1991). "Parallel tempering." Computing Science and Statistics: Proceedings of the 23rd SymComputing Science and S, American Statistical Association, 156. 13

Girolami, M. (2008). "Bayesian inference for differential equations." Theoretical Computer Science, 408: 4-16. 13, 14

Gordon, N. J., Salmond, D. J., and Smith, A. F. M. (1993). "Novel approach to nonlinear/non-Gaussian Bayesian state estimation." IEEE Proceedings F: Radar and Signal processing, 140: 170-113. 11

Lebreton, J. D., Burnham, K. P., Clobert, J., and Anderson, D. R. (1992). "Modeling survival and testing biological hypotheses using marked animals: a unified approach with case studies." Ecological Monographs, 61(1): 67-118. 9

Marzolin, G. (1988). "Polygynie du cincle plongeur (cinclus cinclus) dans le côtes de Loraine." Oiseau et la Revue Francaise d'Ornithologie, 58(4): 277-286. 9

Nott, D. J., Drovandi, C. C., Mengersen, K., and Evans, M. (2016). "Approximation of Bayesian predictive p-values with regression ABC." To appear in Bayesian Analysis. URL http://projecteuclid.org/euclid.ba/1479286819 9

Raue, A., Kreutz, C., Maiwald, T., Bachmann, J., Schilling, M., Klingmüller, U., and Trimmer, J. (2009). "Structural and practical identifiability analysis of partially observed dynamical models by exploiting the profile likelihood." Bioinformatics, 25(15): 1923-1929. 14

Turchin, P. (2003). Complex population dynamics. Princeton University Press. 11 
Wood, S. N. (2010). "Statistical inference for noisy nonlinear ecological dynamic systems." Nature, 466: 1102-1107. 10, 11

Zhou, Y., Johansen, A. M., and Aston, J. A. D. (2016). "Towards automatic model comparison: an adaptive sequential Monte Carlo approach." Journal of Computational and Graphical Statistics, 25(3): 701-726. 20 